\title{
Relationships between Weight, Adiposity, Functional Status, and Left Ventricle Characteristics in Overweight and Obese Patients with Heart Failure
}

\author{
Marjan Motie $^{1 *}$, Lorraine S. Evangelista ${ }^{1}$, Dawn Lombardo², Tamara B. Horwich ${ }^{3}$, Michele Hamilton ${ }^{4}$, \\ Gregg C. Fonarow ${ }^{5}$
}

${ }^{1}$ Program in Nursing Science, University of California Irvine, Irvine, California, USA

${ }^{2}$ Department of Medicine, University of California Irvine Medical Center, Irvine, California, USA

${ }^{3}$ University of California at Los Angeles, David Geffen School of Medicine, Los Angeles, California, USA

${ }^{4}$ Department of Medicine/Cardiology UCLA and Director of the Heart Failure Program, Cedars-Sinai Heart Institute, Los Angeles, California, USA

${ }^{5}$ Director, UCLA Cardiomyopathy Center and Professor of Cardiology, David Geffen School of Medicine, Los Angeles, California, USA

*Corresponding author: Marjan Motie, $\mathrm{PhD}$, Assistant Professional Researcher, UCI Program in Nursing Science, Irvine, CA 92697, California, USA, Tel: (949) 824-8707/ (714) 393-9292; E-mail: mmotie@hs.uci.edu

\begin{abstract}
Aims: This study was conducted to examine the relationship between adiposity and functional status (i.e. peak oxygen consumption [ $\left.\mathrm{VO}_{2} \max \right]$ ), and left ventricular (LV) structural characteristics (i.e., LV ejection fraction [LVEF], LV end diastolic dimension [LVEDD], LV posterior wall thickness [LVPWT]) in heart failure (HF) patients with diabetes mellitus (DM), and/or metabolic syndrome (MS). We hypothesize that excess weight and body fat are significantly related to cardiac functional status.

Methods and Results: Ninety four patients' clinical characteristics were analyzed at baseline to examine the relationships of interest. Results show that weight was correlated with fat and lean mass and LVEF (all p's $<0.050$ ). Novel findings from our data showed that weight, fat mass, and percent fat were inversely related to $\mathrm{VO}_{2}$ max; weight, fat mass and lean mass were positively related with LVPWT. In a multivariate analysis, body mass index and fat mass accounted for $28.8 \%$ of the variance in $\mathrm{VO}_{2}$ max, showing significantly higher predictive value than other covariates $(\mathrm{P}=0.002)$. Conclusions: Our findings show a possible relationship between body fat on functional status in this patient cohort and challenges existing research that supports that higher weight and increased fat are good in the setting of chronic HF (i.e. obesity paradox). Strategies to optimize weight and reduce adiposity warrants further investigation in this subgroup of patients.
\end{abstract}

Keywords: Heart failure, Functional status, Left ventricular characteristics, Obesity
Received Date: September 08, 2016

Accepted Date: February 13, 2017

Published Date: February 20, 2017

Citation: Motie, M., et al. Relationships between Weight, Adiposity, Functional Status, and Left Ventricle Characteristics in Overweight and Obese Patients with Heart Failure. (2017) J diab Obes 4(2): 1-5.

DOI: $10.15436 / 2376-0494.17 .1108$

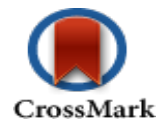

\section{Introduction}

Chronic heart failure (HF) is a healthcare epidemic characterized by progressive decline of cardiac performance and continues to be a public health problem affecting over 5 million Americans $^{[1]}$. Chronic HF is associated with significant comorbidities and frequent decompensation, resulting in recurrent hospitalizations ${ }^{[2,3]}$. Being overweight or obese are well established comorbidities and/or risk factors that lead to health consequences including coronary heart disease, hypertension, diabetes mellitus (DM), dyslipidemia, sleep apnea, and certain cancers ${ }^{[4]}$.

Obesity has been reported as affecting the cardiovascular system directly in many ways, in addition to the indirect consequences caused by the deleterious effects of the known comorbidities and their associated risk factors ${ }^{[5]}$. Many human and animal studies have led to the understand- ing that obesity affects the structure and function of the heart mainly by causing increased blood volume, elevated cardiac output, left ventricular (LV) hypertrophy, and LV diastolic dysfunction all of which also play a role in causing $\mathrm{HF}^{[6]}$.

Recent literature has described the relationship between obesity and overweight with several obesity related complications (e.g., cardiac functional status, LV characteristics) ${ }^{[7]}$; however, these relationships are even less well defined in patients with HF and multiple co-morbidities (e.g., obesity, DM, metabolic syndrome [MS]). Prior research has not been able to distinctly identify the effects of obesity from the effects of the comorbidities which on their own cause LV dysfunction. Furthermore, despite the known deleterious effects of excess body weight as an independent risk factor for cardiovascular disease (CVD), there are multiple studies showing improved survival in obese patients with known CVD (concept known as obesity par- 
$\operatorname{adox})^{[8,9-13]}$. The specific aims of this investigation are to: 1) examine the association between overall weight, body composition (i.e., fat mass, lean mass, percent body fat), and structural and functional cardiac status in this sample of HF patients; and 2) identify independent predictors of functional status to gain some insight into the possible nature of the deleterious vs. beneficial effects of obesity in HF.

\section{Methods}

The enrollment criteria and study design have been described elsewhere ${ }^{[14]}$. Briefly, 94 participants were recruited and provided informed consent to participate in a randomized controlled trial for overweight and obese patients with NYHA Functional Class II-IV HF, DM, and/or MS, irrespective of LVEF (Table 1). The University of California Los Angeles and University of California Irvine Institutional Human Subjects Review Committees approved the study. The investigation conformed with the principles outlined in the Declaration of Helsinki.15 Participants who met the inclusion/exclusion criteria received a 3-month behavioral weight management intervention based on one of two different macronutrient content energy-restricted meal plans (at 1200 or $1500 \mathrm{kcal} /$ day) which would provide a calorie deficit aimed at $500 \mathrm{kcal}$ or more. For the purpose of this descriptive study, weight, adiposity (i.e., fat mass, lean mass, percent body fat), peak oxygen consumption $\left[\mathrm{VO}_{2} \max \right]$ ) -as measured by cardiopulmonary exercise test (CPX) - and LV structural characteristics (i.e. LV ejection fraction [LVEF], LV end diastolic dimension [LVEDD], LV posterior wall thickness [LVPWT] -as measured by echocardiogram- were described for all participants at baseline.

Data was analyzed using SPSS version 19.0 for Windows. Sociodemographic and clinical variables were computed using descriptive statistics (e.g., means and standard deviations for continuous variables and 2 tests for categorical variables). Relationships between variables of interest (weight, body mass index [BMI], fat mass, lean mass, fat $\%, \mathrm{VO}_{2}$ max, LVEF, LVEDD, and LVPWT) were analyzed using Pearson Moment correlations. Multivariate analyses were performed to test the independent association between cardiac function (as measured by $\mathrm{VO}_{2}$ max) and adiposity and BMI (the dependent variables) with age, gender, New York Heart Association (NYHA) class, history of diabetes or hypertension as covariates in one model and the same covariates plus LVEF and LVPWT in another model.

\section{Results}

\section{Participant characteristics}

Table 1 shows the sociodemographic and clinical characteristics of the study sample. Participants ranged in age from 27 to 81 and were on the average moderately obese (BMI, 37.08 $\pm 6.18 \mathrm{~kg} / \mathrm{m}^{2}$, range 27 to 61 ). Participants' baseline clinical characteristics and cardiac structural and functional measurements are also shown in Table 1.

Table 1: Sociodemographic and Clinical Characteristics $(\mathrm{N}=94)$.

\begin{tabular}{|l|l|}
\hline & Baseline All Subjects \\
\hline Age, years (Mean $\pm \mathrm{SD})$ & $58.79 \pm 9.95$ \\
\hline Male (\%) & $69.6 \%$ \\
\hline White (\%) & $65.2 \%$ \\
\hline History of Diabetes & $26.6 \%$ \\
\hline History of Hypertension & $43.6 \%$ \\
\hline History of Smoking & $56.2 \%$ \\
\hline History of Statin use & $68.7 \%$ \\
\hline NYHA class, N (\%) & \\
\hline \multicolumn{1}{|c|}{ Class 2 } & $81.1 \%$ \\
\hline \multicolumn{1}{|c|}{ Class 3 } & $18.9 \%$ \\
\hline Weight (lbs) & $239.63 \pm 46.78$ \\
\hline Body Mass Index (BMI) & $37.08 \pm 6.18$ \\
\hline Percent Fat & $38.06 \pm 7.44$ \\
\hline VO max (ml/kg/min) & $12.31 \pm 3.79$ \\
\hline LVEF (\%) & $39.06 \pm 13.65$ \\
\hline LVDD (mm) & $58.24 \pm 10.58$ \\
\hline LVPWT (mm) & $10.82 \pm 2.21$ \\
\hline
\end{tabular}

$\mathrm{VO}_{2}$ max - peak oxygen consumption; LVEF - left ventricular ejection fraction; LVEDD - left ventricular end diastolic dimension; LVPTW left ventricular posterior wall thickness

\section{Association between weight and body composition and car- diac function}

The correlation matrix for key variables is illustrated in Table 2. As shown, weight and BMI were correlated with fat and lean mass and LVEF (all p's < 0.050). Weights, BMI, fat mass and percent fat were inversely related to $\mathrm{VO}_{2}$ max; while weight and lean mass were positively related with LVPWT.

Table 2: Correlational Matrix of Key Variables of Interest at Baseline ( $\mathrm{N}=94)$.

\begin{tabular}{|c|c|c|c|c|c|c|c|c|c|c|}
\hline Variable & 1 & 2 & 3 & 4 & 5 & 6 & 7 & 8 & 9 & \\
\hline 1. & BMI & 1.000 & & & & & & & & \\
\hline 2. & Body weight (lbs.) & $0.816 \dagger$ & 1.000 & & & & & & & \\
\hline 3. & Fat mass $(\mathrm{g})$ & $0.859 \dagger$ & $0.702 \dagger$ & 1.000 & & & & & & \\
\hline 4. & Lean mass (g) & $0.236^{*}$ & $0.712 \dagger$ & 0.038 & 1.000 & & & & & \\
\hline 5. & Total $\%$ fat & $0.561 \dagger$ & 0.152 & $0.787 \dagger$ & $-0.565 \dagger$ & 1.000 & & & & \\
\hline 6. & $\mathrm{VO}_{2} \max$ & $-0.365 \dagger$ & $-0.221 *$ & $-0.419 \dagger$ & 0.177 & $-0.455 \dagger$ & 1.000 & & & \\
\hline 7. & LVEF (\%) & $0.277^{*}$ & $0.235^{*}$ & 0.209 & 0.023 & 0.123 & 0.035 & 1.000 & & \\
\hline 8. & LVEDD & -0.025 & 0.051 & -0.050 & 0.033 & -0.048 & -0.153 & $-0.622 \dagger$ & 1.000 & \\
\hline 9. & LVPWT & 0.242 & $0.460 \dagger$ & 0.108 & $0.569 \dagger$ & -0.211 & 0.021 & -0.022 & -0.010 & 1.000 \\
\hline
\end{tabular}

$* \mathrm{P}<.05, \dagger \mathrm{P}<.001$ 


\section{Subgroup Analysis}

The multivariate analyses showed that the variance in the outcome, $\mathrm{VO}_{2}$ max, explained by all the predictors in a model with age, gender, NYHA class, history of diabetes or hypertension is not different from a model with any of the predictors $(\mathrm{F}$ $(5,39)=1.97, \mathrm{P}=0.105)$ as shown in Table 3 , model 1 . Table 3 , model 2 illustrates that sequentially adding LVPWT and LVEF to the list of predictors shows no further contribution to explaining the variance in $\mathrm{VO}_{2} \max (\mathrm{F}(2,37)=1.175, \mathrm{P}=0.320)$. In model 3, the subsequent addition of BMI and fat as predictors improves the previous models significantly $(\mathrm{P}=0.007)$. Overall, fat mass along with BMI accounted for $28.8 \%$ of the variance in $\mathrm{VO}_{2}$ max while the cardiac structural measures (LVEF and LVPWT) had much lower predictive correlations (10.7\%).

Table 3: Multivariate Analysis of Variables.

\begin{tabular}{|l|c|c|c|c|c|}
\hline & & & \multicolumn{3}{|c|}{ Change Statistics } \\
\hline & Adjusted R- Squared & Std Error of the Estimate & R square Change & F Change & Significant F Change \\
\hline Model 1 & 0.099 & 3.59 & 0.202 & 1.97 & 0.105 \\
\hline Model 2 & 0.107 & 3.57 & 0.048 & 1.18 & 0.320 \\
\hline Model 3 & 0.288 & 3.19 & 0.185 & 5.71 & 0.007 \\
\hline
\end{tabular}

Predictors: Model 1: Age, gender, New York Heart Association Class (NYHA), history of hypertension and diabetes; Model 2: Age, gender, NYHA Class, history of hypertension and diabetes, LVEF and LVPWT; Model 3: Age, gender, NYHA Class, history of hypertension and diabetes, LVEF, LVPWT, FAT (gram) and BMI

Testing: $\mathrm{VO}_{2} \max$

\section{Discussion}

To our knowledge, this is the first study to explore the relationship between adiposity and cardiac structure and function in overweight and obese patients with HF who also have DM and/or MS. Although the higher incidence of CVD in obese individuals has been extensively reported as being linked to known risk factors such as dyslipidemia, hypertension, glucose intolerance, inflammatory markers, and obstructive sleep apnea, ${ }^{[5,6,16]}$ the relationship between obesity and the structural and functional characteristics of the heart itself has not been fully explored in this population.

Several studies have recently described the possible effects of obesity on the left ventricle ${ }^{[16-19]}$. However, the pathogenesis of LV dysfunction in obesity is not completely understood but has been linked to an increase in plasma volume and long term elevation in cardiac output (as characterized by dilated, hypertrophied ventricles and increased stroke volume), both resulting from the amplified metabolic demands of both excessive fatty tissue and lean body mass ${ }^{[5,6]}$. Obesity was reported as being associated with concentric LV remodeling with no accompanying change in ejection fraction in a large population based study ${ }^{[18]}$. In obese adolescent girls, the onset of cardiac dysfunction and the extent to which LV size, stroke volume and cardiac output increased were reported to be related to the severity of obesity ${ }^{[20]}$. In HF patients, Lavie and colleagues ${ }^{[21]}$ concluded that there were no significant differences in LV ejection phases between lean and obese patients (independent of HF etiology).

Considering the known adverse effects of obesity on LV structure and function, the findings of a recent report that demonstrated a positive correlation between BMI and worse diastolic function in a community based elderly cohort comes as no surprise ${ }^{[17]}$. This relationship was discussed as the possible reason for putting overweight and obese persons at increased risk for new onset HF since LV dysfunction is recognized as a possible pathophysiological link in the etiology of obesity induced HF. On the other hand, many studies have reported that once HF is established, obese individuals have a better overall clinical prognosis and reduced risk of mortality (referred to as the obesity paradox in HF) ${ }^{[1,12,13,22,23]}$. Our findings show that
BMI is positively correlated with LVEF. These results appear to be in agreement with the studies describing an apparent obesity paradox in terms of the overall better LV diastolic function in more obese HF patients. Our results further showed a positive correlation between LVEF and LVEDD (as expected) but no correlation between the latter and fat or lean mass or BMI. Although the LV dilation is not affected by increased weight, the LV weight (measured by LVPWT) was significantly correlated with weight and lean mass and not fat mass. This parameter is known to measure the muscle mass surrounding the heart such that lower lean mass would be expected to contribute to lower heart muscle mass. In the study of obese subjects done at necropsy ${ }^{[22,24]}$, an increase in heart weight, wall thickness and LV hypertrophy were observed; however, while the mechanism of LV hypertrophy was once attributed to excess body fat, recent data has attributed fat-free mass as a stronger predictor of LV mass and LV hypertrophy ${ }^{[22,25]}$.

Despite the favorable change in cardiac diastolic function with excess body weight, the significant negative relationship between fat mass (and $\mathrm{BMI}$ ) and $\mathrm{VO}_{2}$ max (the gold standard in the assessment of clinical status in HF) is a novel finding that supports our hypothesis that excess fat may not be protective in HF patients ${ }^{[26]}$. Furthermore, the deleterious association of excess weight on $\mathrm{VO}_{2}$ max was not significantly correlated with any of the cardiac structural and functional parameters.

Previous studies of CPX in HF have only identified a link to the etiology of HF where prognosis was improved in ischemic obese or overweight HF patients whereas non-ischemic patients ${ }^{[11,27]}$ had similar outcomes as normal weight patients. It has also been reported that the cardiorespiratory fitness (FIT) of HF patients is an important factor in the relationship between obesity and prognosis; those with high FIT (peak $\mathrm{VO}_{2}>14 \mathrm{~mL} \mathrm{O}_{2} \cdot \mathrm{kg}^{-1}$ - $\left.\min ^{-1}\right)$, demonstrated good prognosis with no evidence of an obesity paradox, but also no evidence that obesity was associated with worse outcomes ${ }^{[9,21]}$. In another report, researchers found that particularly in women and obese patients, who generally have a higher body fat content, peak $\mathrm{VO}_{2}$ best estimates exercise capacity (as a prognostic factor in survival) when it is computed by dividing by lean mass (rather than total weight) ${ }^{[28]}$. This was expressed as being related to the importance of skeletal muscles 
in the pathophysiology of HF. Our findings from a multivariate analysis show that the difference in $\mathrm{VO}_{2}$ max among overweight and obese HF patients is best explained by fat mass (and BMI) as the dependent variable while keeping other risk factors constant (gender, age, NYHA class, history of hypertension and diabetes, LVEF, and LVPWT). To the best of our knowledge, this is the first report of the relationship of $\mathrm{VO}_{2}$ max and body composition in this population suggesting that intentional weight loss may potentially result in better outcomes particularly for those with lower $\mathrm{VO}_{2}$ max.

The present report shows a trend toward worsening cardiac function with increasing weight in HF patients with DM or MS. We did not have a concurrent control group of overweight or obese patients with no HF etiology. Also, there were very few patients with more advanced HF (NYHA class 4 ) to delineate the associations of worsening disease versus associations of increasing weight. Finally, another limitation of the current study was the small sample size.

The results of this study provide evidence that greater fat mass in obese patients with HF along with DM, and/or MS is an independent predictor of worse functional capacity of the heart. Future studies are warranted to examine the effect of weight management interventions (and differentiating between different approaches to fat loss) on the changes associated with cardiac status over short and long term duration in this highly vulnerable population.

Acknowledgements and Funding: This work was supported by funding from the National Heart, Lung, and Blood Institute (1R01HL093466-01). The content is solely the responsibility of the authors and does not necessarily represent the official views of the National Heart, Lung, and Blood Institute-National Institutes of Health. The authors would like to acknowledge support from the UCI Institute for Clinical Translational Science (ICTS) and UCLA Clinical and Translational Science Institute (CTSI). The project described was supported by the National Institutes of Health / National Center for Research Resources and the National Center for Advancing Translational Sciences (NCATS), through UCI ICTS Grant number UL1 TR000153 and by NIH/ NCATS, UCLA CTSI Grant Number UL1TR000124. The content is solely the responsibility of the authors and does not necessarily represent the official views of the NIH.

Conflict of Interest: None declared

\section{References}

1. Lloyd-Jones, D., Adam, R.J., Brown, T.M. Executive summary: heart disease and stroke statistics--2010 update: a report from the American Heart Association. (2010) Circulation 121: e215.

Pubmed

2. Chun, S., Tu, J.V., Wijeysundera, H.C. et al. Lifetime analysis of hospitalizations and survival of patients newly-admitted with heart failure. (2012) Circ Heart Fail 5(4): 414-421.

Pubmed | Crossref

3. Desai, A.S., Stevenson, L.W. Rehospitalization for Heart Failure: Predict or Prevent? (2012) Circulation 126(4): 501-506.

Pubmed | Crossref

4. Mokdad, A.H., Bowman, B.A., Ford, E.S., et al. The continuing epidemics of obesity and diabetes in the United States. (2001) JAMA 286: 1195-1200.

Pubmed | Crossref

5. Mathew, B., Francis, L., Kayalar, A. Obesity: Effects on Cardiovascular Disease and its Diagnosis. (2008) Journal of the American Board of Family Medicine 21: 562-568.

Pubmed | Crossref

6. Poirier, P., Giles, T.D., Bray, G.A. et al. Obesity and Cardiovascular Disease: Pathophysiology, Evaluation, and Effect of Weight Loss: An Update of the 1997 American Heart Association Scientific Statement on Obesity and Heart Disease From the Obesity Committee of the Council on Nutrition, Physical Activity, and Metabolism. (2006) Circulation 113: 898-918.

Pubmed | Crossref

7. Lavie, C.J., McAuley, P.A., Church, T.S., et al. Obesity and cardiovascular diseases: implications regarding fitness, fatness, and severity in the obesity paradox. (2014) J Am Coll Cardiol 63: 1345-1354.

Pubmed | Crossref

8. Clark, A.L., Fonarow, G.C., Horwich, T.B. Obesity and the obesity paradox in heart failure. (2014) Prog Cardiovasc Dis 56: 409-414.

Pubmed | Crossref

9. Lavie, C.J., De Schutter, A., Parto, P., et al. Obesity and prevalence of cardiovascular diseases and prognosis - The obesity paradox updated. (2016) Prog Cardiovasc Dis 58: 537-547.

Pubmed | Crossref

10. Horwich, T.B., Fonarow, G., Hamilton, M.A., et al. The relationship between obesity and mortality in patients with heart failure. (2001) J Am Coll Cardiol 38: 789-795.

Crossref

11. Arena, R., Lavie, C.J. The obesity paradox and outcome in heart failure: is excess bodyweight truly protective? (2009) Future Cardiol 6: $1-6$.

Pubmed | Crossref

12. Fonarow, G.C., Srikanthan, P., Costanzo, M.R., et al. An obesity paradox in acute heart failure: Analysis of body mass index and inhospital mortality for 108927 patients in the Acute Decompensated Heart Failure National Registry. (2007) American Heart Journal 153: 74-81.

Pubmed | Crossref

13. Lavie, C.J., Mehra, M.R., Milani, R.V. Obesity and heart failure prognosis: paradox or reverse epidemiology? (2005) Eur Heart J 26: 5-7.

Pubmed | Crossref

14. Motie, M., Evangelista, L.S., Horwich, T.B. et al. Pro-HEART a randomized clinical trial to test the effectiveness of a high protein diet targeting obese individuals with heart failure: rationale, design and baseline characteristics. (2013) Contemp Clin Trials 36: 371-381. Pubmed | Crossref

15. Rickham, P.P. Human experimentation. Code of ethics of the world medical association. (1964) Declaration of Helsinki Br Med J 2: 177. PMid:14150898

Pubmed | Crossref 
16. Litwin, S.E. Cardiac Remodeling in Obesity- Time for a New Paradigm. (2010) JACC 3: 275-277.

Pubmed | Crossref

17. Russo, C., Jin, Z., Homma, S., et al. Effect of Obesity and Overweight on Left Ventricular Diastolic Function: A Community-Based Study in an Elderly Cohort. (2011) JACC 57: 1368-1374.

Pubmed | Crossref

18. Turkbey, E.B., McClelland, R.L., Kronmal, R.A., et al. The Impact of Obesity on the Left Ventricle. The Multi-Ethnic Study of Atherosclerosis (MESA). (2010) JACC 3: 266-274.

Pubmed | Crossref

19. Alpert, M.A., Omran, J., Mehra, A., et al. Impact of obesity and weight loss on cardiac performance and morphology in adults. (2014) Prog Cardiovasc Dis 56: 391-400

Pubmed | Crossref

20. Rowland, T.W., Dunbar, N.S. State of the Art Reviews: Effects of Obesity on Cardiac Function in Adolescent Females. (2007) American Journal of Lifestyle Medicine 1: 283-288.

Crossref

21. Lavie, C.J., Cahalin, L.P., Chase, P., et al. Impact of Cardiorespiratory Fitness on the Obesity Paradox in Patients With Heart Failure. (2013) Mayo Clin Proc 88: 251-258.

Pubmed | Crossref
22. Lavie, C.J., Alpert, M.A., Arena, R., et al. Impact of Obesity and the Obesity Paradox on Prevalence and Prognosis in Heart Failure. (2013) JACC: Heart Failure 1: 93-102.

Pubmed | Crossref

23. Kalantar-Zadeh, K., Anker, S.D., Coats, A.J.S., et al. Obesity Paradox as a Component of Reverse Epidemiology in Heart Failure. (2005) Archives of Internal Medicine 165: 1797.

Pubmed | Crossref

24. Warnes, C.A., Roberts, W.C. The heart in massive (more than 300 pounds or 136 kilograms) obesity: Analysis of 12 patients studied at necropsy. (1994) Am J Cardiol 54: 1087-1091.

Pubmed | Crossref

25. Bella, J.N., Devereux, R.B., Roman, M.J., et al. Relations of left ventricular mass to fat free and adipose body mass: the Strong Heart Study. (1998) Circulation 98: 2538-2544.

Pubmed | Crossref

26. Lavie, C.J., Milani, R.V., Ventura, H.O. Obesity and cardiovascular disease: risk factor, pradox, and impact of weight loss. (2009) J Am Coll Cardiol 53: 1925-1932.

Pubmed | Crossref

27. Arena, R., Myers, J., Abella, J., et al. Influence of etiology of heart failure on the obesity paradox. (2009) Am J Cardiol 104: 1116-11121. Pubmed | Crossref

28. Cicoira, M., Davos, C.H., Francis, D.P., et al. Prediction of mortality in chronic heart failure from peak oxygen consumption adjusted for either body weight or lean tissue. (2004) Journal of Cardiac Failure 10: 421-426.

Pubmed | Crossref
Ommega Online Publishers

Journal Title: Journal of Diabetes and Obesity (JDO)

Journal Short Name: J diabetes Obes
Journal ISSN: 2356-0494

E-mail: diabetes@ommegaonline.com

Website: www.ommegaonline.org 